OPEN ACCESS

Edited by:

Thomas Dandekar,

University of Würzburg, Germany

Reviewed by:

Peter Mullany,

University College London,

United Kingdom

Eugenie Huillet,

INRA Centre Jouy-en-Josas, France

*Correspondence:

Simon M. Cutting

s.cutting@rhul.ac.uk

Paula S. Salgado

paula.salgado@ncl.ac.uk

tThese authors have contributed

equally to this work.

Specialty section:

This article was submitted to

Infectious Diseases,

a section of the journal

Frontiers in Microbiology

Received: 23 May 2017 Accepted: 05 September 2017 Published: 21 September 2017

Citation:

Dembek M, Willing SE, Hong HA, Hosseini S, Salgado PS and

Cutting SM (2017) Inducible Expression of spoOA as a Universal Tool for Studying Sporulation

in Clostridium difficile.

Front. Microbiol. 8:1793.

doi: 10.3389/fmicb.2017.01793

\section{Inducible Expression of spoOA as a Universal Tool for Studying Sporulation in Clostridium difficile}

\author{
Marcin Dembek ${ }^{11}$, Stephanie E. Willing ${ }^{2 t}$, Huynh A. Hong ${ }^{2}$, Siamand Hosseini2, \\ Paula S. Salgado ${ }^{*}$ and Simon M. Cutting ${ }^{2 *}$ \\ ${ }^{1}$ Institute for Cell and Molecular Biosciences, Newcastle University, Newcastle upon Tyne, United Kingdom, ${ }^{2}$ School of \\ Biological Sciences, Royal Holloway, University of London, London, United Kingdom
}

Clostridium difficile remains a leading nosocomial pathogen, putting considerable strain on the healthcare system. The ability to form endospores, highly resistant to environmental insults, is key to its persistence and transmission. However, important differences exist between the sporulation pathways of $C$. difficile and the model Grampositive organism Bacillus subtilis. Amongst the challenges in studying sporulation in C. difficile is the relatively poor levels of sporulation and high heterogeneity in the sporulation process. To overcome these limitations we placed $P_{\text {tet }}$ regulatory elements upstream of the master regulator of sporulation, spoOA, generating a new strain that can be artificially induced to sporulate by addition of anhydrotetracycline (ATC). We demonstrate that this strain is asporogenous in the absence of ATc, and that ATc can be used to drive faster and more efficient sporulation. Induction of SpoOA is titratable and this can be used in the study of the SPOOA regulon both in vitro and in vivo, as demonstrated using a mouse model of $C$. difficile infection (CDI). Insights into differences between the sporulation pathways in $B$. subtilis and $C$. difficile gained by study of the inducible strain are discussed, further highlighting the universal interest of this tool. The

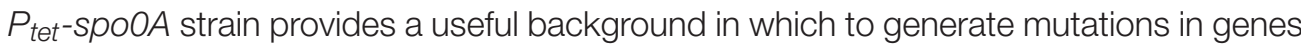
involved in sporulation, therefore providing an exciting new tool to unravel key aspects of sporulation in C. difficile.

\footnotetext{
Keywords: sporulation, Clostridium difficile, spo0A, spore, inducible expression, allele-coupled exchange, anhydrotetracycline
}

\section{INTRODUCTION}

Clostridium difficile is a Gram-positive, anaerobic, spore-forming pathogen and the leading cause of healthcare associated diarrhea worldwide (Rupnik et al., 2009; Smits et al., 2016). C. difficile infection (CDI) occurs most commonly in nosocomial settings, among patients whose natural gut microbiota has been perturbed by prolonged treatment with broad-spectrum antibiotics (Dethlefsen et al., 2008; Lawley and Walker, 2013). Two large cytotoxins secreted by the vegetative cell are responsible for the clinical manifestations of CDI, which range from mild, self-limiting diarrhea to severe, often fatal inflammatory complications, such as pseudomembranous colitis or toxic megacolon (Dubberke and Olsen, 2012). However, it is the spore that is the primary infectious agent, as mutant strains defective in sporulation are unable to efficiently persist in the environment and transmit disease (Deakin et al., 2012). Due to their multi-layered structure, spores are extremely robust and resistant to both chemical and physical insults, enabling C. difficile to survive exposure to heat, oxygen, alcohol, noxious chemicals, and certain disinfectants 
(Lawley et al., 2010; Vohra and Poxton, 2011). Despite its key relevance in CDI, many aspects of sporulation in C. difficile are still understudied, hampered by low sporulation efficiency and high heterogeneity in the sporulation process.

In order to sporulate, the cell must undergo an ordered program of unicellular differentiation that leads to a dormant, non-replicative state. The decision to enter the developmental program is highly regulated, requiring multiple environmental and metabolic cues (Al-Hinai et al., 2015). It is mediated by Spo0A, a conserved transcription factor that plays a central role in phenotypic differentiation and adaptation to changing environments. The role of Spo0A in orchestrating sporulation has been studied in exquisite detail in Bacillus subtilis (reviewed in Sonenshein, 2000). Spo0A is post-translationally activated via phosphorylation facilitated by a complex phosphorelay consisting of several sensor kinases (KinA-E). These kinases respond to external signals by auto-phosphorylating and subsequently transferring a phosphate residue either directly to Spo0A or via intermediary proteins Spo0F and Spo0B (Jiang et al., 2000b). In contrast to B. subtilis, there are no Spo0F or Spo0B homologs, and therefore presumably no phosphorelay system, in C. difficile; rather, Spo0A is believed to be phosphorylated directly by the sensor kinases (Underwood et al., 2009; Steiner et al., 2011).

The phosphorelay affords opportunity for multiple layers of regulation, such as Rap phosphatases that modulate the level of phosphorylated SpoOA, permitting a degree of fine-tuning and flexibility to the system (Veening et al., 2005). It remains unclear whether this level of control is absent in C. difficile or is achieved by other means (Jiang et al., 2000a; Paredes et al., 2005). Studies published to date have shown that $C$. difficile initiates sporulation in response to nutrient availability (Edwards et al., 2014; Nawrocki et al., 2016) and that a Rap-like protein, RtsA, acts as a positive regulator of sporulation, although the exact nature of this effect remains unclear (Edwards et al., 2016). Sporulation, initiated by an asymmetric division of the vegetative cell into a smaller forespore and a larger mother cell, then proceeds via an expression cascade controlled by sporulation-specific sigma factors $\left(\sigma^{\mathrm{E}}, \sigma^{\mathrm{F}}, \sigma^{\mathrm{G}}\right.$, and $\left.\sigma^{\mathrm{K}}\right)$. Differential expression of sigma factors in each of those compartments allows the sporulation process to be completed.

Recent studies of Spo0A in B. subtilis have demonstrated that the importance of this regulator extends beyond just sporulation, revealing the existence of an intricate Spo0A regulatory network fine-tuned to respond to the specific amount of Spo0A present in the cell (Fujita et al., 2005; Vishnoi et al., 2013). Efficient sporulation requires a progressive increase in the levels of Spo0A, which prompts the gradual activation initially of low-threshold Spo0A-regulated genes and subsequently high-threshold Spo0Aregulated genes (Fujita et al., 2005; Vishnoi et al., 2013). In B. subtilis the Spo0A regulon comprises approximately 120 genes (Molle et al., 2003). At low levels of Spo0A, genes involved in lifestyle changes that might delay the need to sporulate, such as biofilm formation, are activated and only if levels of Spo0A continue to rise until a threshold level is reached does the cell commit to sporulation (Fujita et al., 2005). Thus, Spo0A serves as a focal point for the integration of a number of factors that determine whether the cell needs to enter a dormant state. In C. difficile, Spo0A is thought to control approximately 300 genes (Fimlaid et al., 2013; Pettit et al., 2014), many of which have been linked to biofilm formation (Dawson et al., 2011), swimming motility (Pettit et al., 2014) and toxin production (Underwood et al., 2009), although conflicting results have been reported across different strains and laboratories (Rosenbusch et al., 2012; Mackin et al., 2013), indicating the complex nature of Spo0A signaling.

In an attempt to provide a more robust and tractable way to study sporulation in $C$. difficile, we have generated an inducible spo0A strain and investigated whether controlled expression of the transcription factor is a viable method for achieving higher sporulation efficiency and homogeneity. We demonstrate that spo0A expression can be induced in a dose-dependent manner and that the level of expression correlates with sporulation efficiency, which reaches values higher than those observed in an isogenic wild type (WT) strain. We also show that spo0Adependent genes involved in other aspects of the bacterial life cycle can be indirectly controlled by use of the inducing agent. Finally, using the mouse model of CDI, we demonstrate that induction of $s p o 0 A$ can be carried out in vivo, providing a new tool in studying the role of Spo0A-dependent phenotypes in virulence.

\section{RESULTS}

\section{Generating an Inducible spo0A Strain}

Allele-coupled exchange was used to place the inducible $P_{\text {tet }}$ promoter with its regulatory elements $30 \mathrm{bp}$ upstream of the spo0A ORF on the C. difficile 630 chromosome, generating

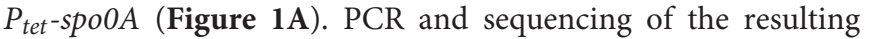
product was used to confirm correct integration of the promoter (Figure 1B). The tet element comprises two divergent promoters, $P_{\text {tet }}$ and $P_{\text {tet }}$, each overlapping with a tet operator sequence and regulated by tetracycline (Figure 1C). TetR, which represses both $P_{\text {tet }}$ and $P_{\text {tetR }}$, introduces a negative feedback loop to the system that is tightly dose-dependent, allowing an increased level of control (Fagan and Fairweather, 2011). To minimize potential confounding factors resulting from using an antibiotic, the non-antibiotic tetracycline analog anhydrotetracycline (ATc) was used as the inducing agent. To exclude the possibility that ATc was exerting a deleterious effect on normal growth, WT and $P_{\text {tet }}$-spo0A cultures were incubated in the presence or absence of ATc and their growth rate was monitored over time by measuring optical density $\left(\mathrm{OD}_{600}\right)$. No appreciable difference in growth rate was observed between WT and $P_{\text {tet }}$-spo0A, irrespective of the ATc concentration used (Supplementary Figure S1).

\section{Expression of spoOA and spo0A-Dependent Genes in $P_{\text {tet }}$-spo0A Can be Controlled in Response to ATC}

To verify whether spo0A expression can be driven by the introduced $P_{\text {tet }}$ promoter upon induction, qRT-PCR was used 


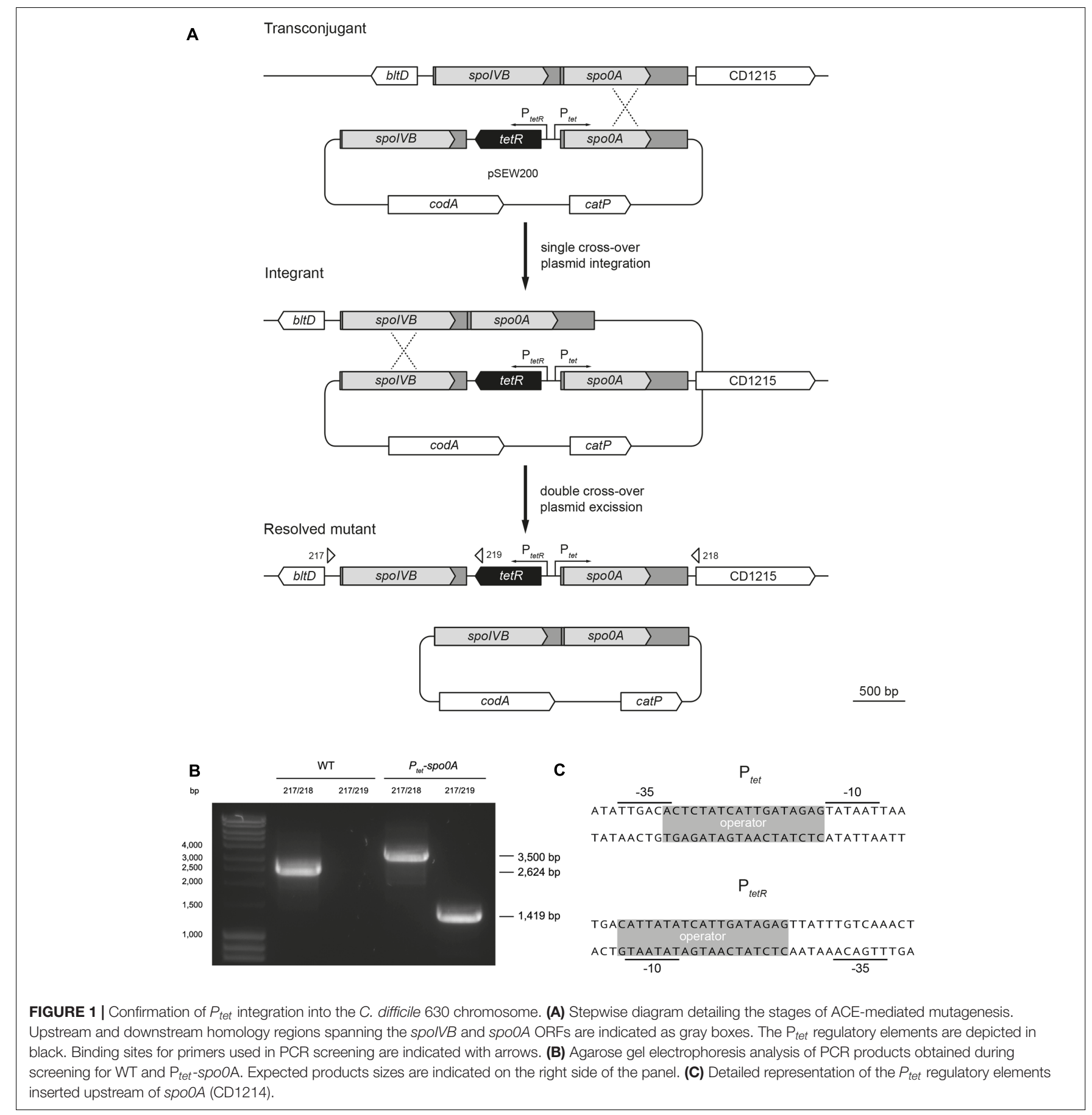

to measure the level of $s p o 0 A$ transcripts in $P_{\text {tet }}-s p o 0 A$ relative to WT following growth with a range of ATc concentrations spanning 0-250 $\mathrm{ng} / \mathrm{ml}$. A clear dose-dependent response in transcript levels was observed (Figure 2A). In the absence of the inducing agent, $P_{\text {tet }}-s p o 0 A$ showed an approximately 40 -fold reduction in detectable spo0A mRNA when compared to WT ( $p<0.0001$ ), a background level of expression possibly attributable to a small amount of promoter 'leakage.' Transcript levels gradually increased with rising ATc concentration, with the largest change observed between 50 and $100 \mathrm{ng} / \mathrm{ml}$, where the levels of spo0A transcripts in $P_{\text {tet }}-s p o 0 A$ were fourfold higher than those found in WT at $100 \mathrm{ng} / \mathrm{ml}$. At the highest concentration of ATc used, $250 \mathrm{ng} / \mathrm{ml}$, spo0A transcript levels were approximately 5.6-fold higher in the mutant relative to WT grown at the same concentration of ATc $(p=0.0161)$ (Figure 2A).

Spo0A is linked to many phenotypes in C. difficile. One of these is flagella-mediated motility where Spo0A has been shown to negatively regulate a number of genes, including 


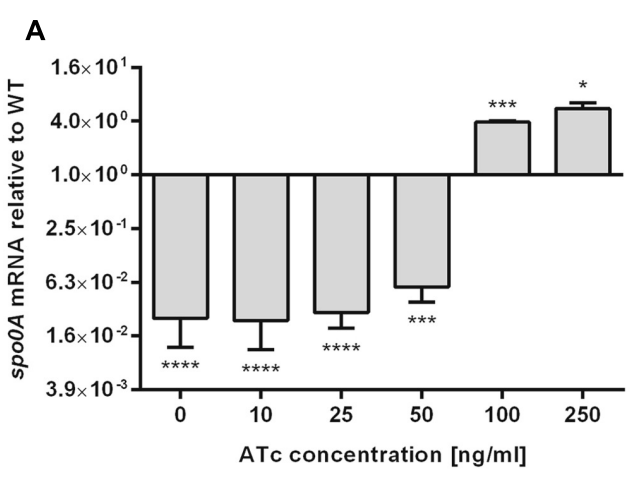

C
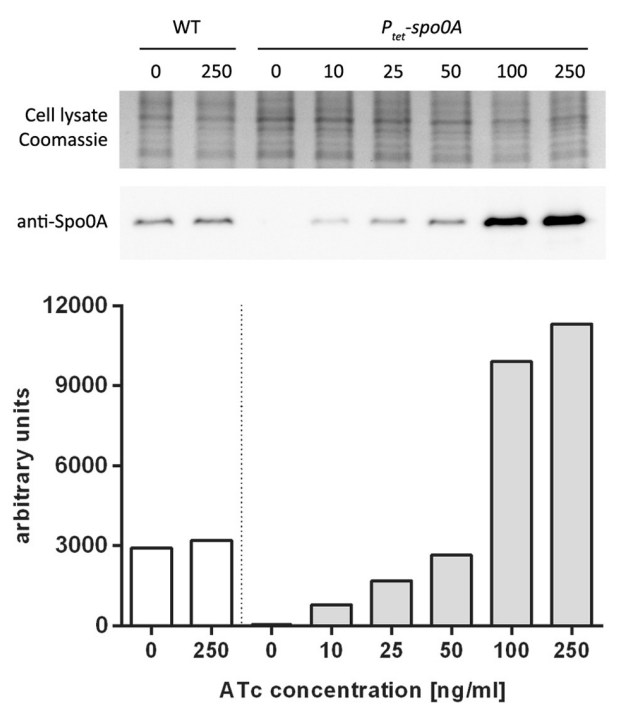

B

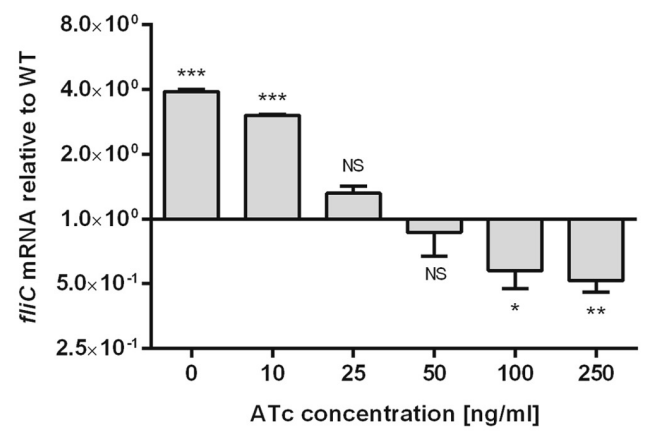

D
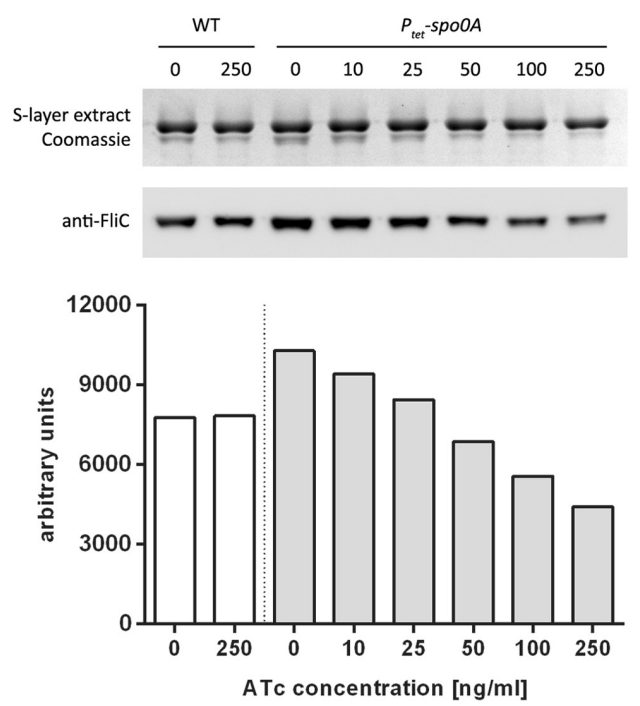

FIGURE 2 | Analysis of dose-dependent expression of $s p \circ 0 \mathrm{~A}$ and fliC in $P_{\text {tet }}-\mathrm{Sp} 00 \mathrm{~A}$. qRT-PCR analysis of spoOA (A) and fliC (B) transcripts isolated from WT or

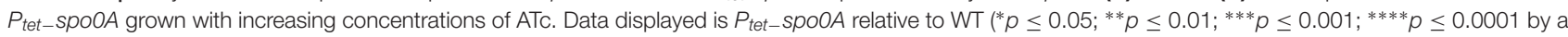
two-tailed Student's $t$-test). Densitometric analysis of anti-SpoOA (C) and anti-FliC (D) immunoblots on protein extracts form WT or $P$ tet-spoOA cells grown with increasing concentrations of ATc. Coomassie-stained gels are provided as loading control.

fliC (Pettit et al., 2014). To further validate the $P_{\text {tet }}$-spo0A system and to demonstrate that the strain can be used to study spo0A-related phenotypes beyond sporulation, we sought to confirm the link between fliC and spo0A by way of ATc induction. In keeping with previous reports on the link between Spo0A and FliC, qRT-PCR demonstrated that $f$ liC transcription levels decreased with increasing ATc concentrations, dropping from 3.9-fold $(p=0.0003)$ to 0.5 -fold $(p=0.0075)$ relative to WT (Figure $2 \mathrm{~B})$. To confirm that the dose-dependent changes in spoOA and fliC transcript levels observed upon ATc induction correlated with similar changes in protein levels, OD-normalized protein extracts from $P_{\text {tet }}$-spo0A cultures grown with a range of ATc concentrations were probed for Spo0A and FliC by Western blotting. In line with our qRT-PCR results, levels of Spo0A in whole cell lysates increased with the increase in ATc concentration while the amount of FliC in surface layer (S-layer) extracts decreased. Importantly, the amount of Spo0A and FliC remained constant in WT, irrespective of ATc concentration (Figures 2C,D).

\section{Sporulation Efficiency in $P_{\text {tet }}$-spo0A Can be Controlled in Response to ATc}

Studies reported to date have shown substantial variation in sporulation between different strains of C. difficile (Underwood et al., 2009; Burns et al., 2010; Vohra and Poxton, 2011). Accordingly, making comparisons between strains has proven problematic and is further compounded by a lack of uniform methods for induction and assessment of sporulation (reviewed in Burns and Minton, 2011). Irrespective of the methodologies used, efficiency of sporulation under laboratory conditions appears to be low, particularly when compared to B. subtilis, hampering work on the molecular basis of sporulation in C. difficile. Having shown that spo0A expression can be manipulated in $P_{t e t}$-spo0A via induction with ATc, we asked whether the observed changes in Spo0A levels would affect the 
overall sporulation efficiency. We first determined whether the tet element is sufficiently tightly regulated to render the $P_{\text {tet }}-s p o 0 A$ strain asporogenous in the absence of ATc. Supporting this, after $120 \mathrm{~h}$ of nutrient starvation in liquid culture, typically regarded as the time after which the sporulation process of a $C$. difficile culture is complete (Burns and Minton, 2011), no spores could be detected based on heat-resistant CFU counts (Figure 3B). As expected, $P_{\text {tet }}$-spo0A cultures grown in the presence of increasing concentrations of ATc showed a dose-dependent increase in heat-resistant CFUs within $16 \mathrm{~h}$ from induction. Maximum spore titers were observed at $24-48 \mathrm{~h}$ post-inoculation, reaching $3 \times 10^{3} \pm 3.28 \times 10^{2} \mathrm{CFU} / \mathrm{ml} ; 2.25 \times 10^{4} \pm 1.32 \times 10^{3} \mathrm{CFU} / \mathrm{ml}$; $1.48 \times 10^{5} \pm 2.47 \times 10^{4} \mathrm{CFU} / \mathrm{ml} ; 5.17 \times 10^{5} \pm 2.75 \times 10^{5}$ $\mathrm{CFU} / \mathrm{ml}$ and $9.83 \times 10^{5} \pm 1.04 \times 10^{5} \mathrm{CFU} / \mathrm{ml}$ in cultures induced with $10,25,50,100$, and $250 \mathrm{ng} / \mathrm{ml}$ of ATc respectively (Figures 3A,C), and remained largely constant throughout the duration of the experiment. Taking into account the total $\mathrm{CFU}$ observed at $\mathrm{t} 0$, these values correspond to a sporulation efficiency of $0.001 \%$ (10 ng/ml ATc), $0.006 \%$ (25 ng/ml ATc), $0.039 \%$ (50 ng/ml ATc), $0.387 \%$ (100 ng/ml ATc), and $1.209 \%$ (250 ng/ml ATc) showing a clear dose response. Importantly the observed titers were reached faster and, at the maximum concentration of ATc, were significantly higher than in WT $\left(2.58 \times 10^{5} \pm 1.25 \times 10^{4} \mathrm{CFU} / \mathrm{ml}\right)(p=0.0364)$ (Figure 3B). While the inducing agent had no effect on the final spore titers observed in WT cultures, it did change the dynamics of the sporulation process, resulting in higher spore titers at 16-24 h post-induction (Figure 3B), possibly an effect of additional stress that high concentration of ATc exerts on the cells.

\section{Controlled Expression of spoOA Has No Effect on Overall Homogeneity of Sporulating Cultures}

The lack of synchronicity observed in sporulating C. difficile cultures has been a significant barrier to population-wide studies as any measurements have to be interpreted as an average value across cells at different stages of the bacterial life cycle. Since sporulation in $P_{t e t}-s p o 0 A$ under ATc induction was capable of proceeding faster than in WT, we asked whether this would be reflected in a more synchronous process. To investigate this, we monitored sporulating $P_{\text {tet }}$-spo0A cultures grown in the presence of increasing ATc concentrations by fluorescence microscopy following staining with FM4-64 and Hoechst 33258 to visualize the membrane and DNA, respectively. In order to overcome the high limit of detection of the technique, cultures were sporulated on a 70:30 mixture of sporulation media (SM) and BHIS agar, previously used in sporulation studies by several groups as it seems to provide faster and more efficient sporulation (Fimlaid et al., 2013; Putnam et al., 2013; Edwards et al., 2016). In line with our previous experiments conducted in liquid culture, a clear dose response in sporulation efficiency was observed, although a twofold higher concentration of ATc had to be used to reach saturation, presumably due to limited ATc availability in plated culture when compared to liquid culture. Mean values of sporulation efficiency, calculated as the percentage ratio of cells that had undergone asymmetric cell division to total cells counted, reached $0.47 \pm 1 \% ; 12.37 \pm 2.35 \% ; 50.44 \pm 6.17 \%$, and $58.99 \pm 6.02 \%$ in cultures induced with $50,100,250$, and $500 \mathrm{ng} / \mathrm{ml}$ ATc, respectively. No sporulation was observed in cultures induced with $25 \mathrm{ng} / \mathrm{ml}$ ATc or in uninduced cultures. As expected, ATc had no significant effect on sporulation efficiency in WT (Figure 4A), reaching $55.6 \pm 3.04 \%$ in the uninduced culture and $55.93 \pm 6.59 \%$ in cultures induced with $500 \mathrm{ng} / \mathrm{ml}$ ATc. In order to assess whether controlled expression of spo0A has an effect on the overall homogeneity of sporulation, we examined the composition of sporulating cultures by counting cells that had reached one of three defined morphological stages: (i) cells with flat, asymmetric septa; (ii) cells undergoing engulfment and (iii) cells with fully engulfed sporangia (including mature spores) (Figure 4B). While the distribution of cells at stage 1,2 , or 3 did change with increasing ATc concentration, the overall homogeneity of the culture did not improve when compared to WT, particularly at higher concentrations of ATc required to match WT sporulation efficiency, suggesting that controlled expression of $s p o 0 A$ on its own does not increase the synchronicity of the sporulating culture (Figures $4 \mathrm{~A}, \mathrm{C}$ ).

\section{Use of $\boldsymbol{P}_{\text {tet-spoOA as a Background }}$ Strain in Studies of Sporulation-Related Phenotypes}

One of the main benefits of using allele-coupled exchange to genetically engineer $C$. difficile is the possibility of creating strains carrying multiple mutations through subsequent rounds of mutagenesis since any changes to the chromosome are left unmarked and therefore not reliant on the limited availability of suitable antibiotic resistance markers (Cartman et al., 2012; Heap et al., 2012). Having shown that inducible expression of $s p o 0 A$ is a viable strategy for controlling sporulation in C. difficile, we set out to investigate whether $P_{t e t}-s p o 0 A$ could be used as a parental strain in studies of sporulation-related genes. As 'proof of concept' we have created sigE, sigF, and sigG mutants in the $P_{\text {tet }}-s p o 0 A$ background by introducing in-frame deletions into the respective genes by allele-coupled exchange. The resulting strains designated $P_{\text {tet }}-s p o 0 A \triangle s i g E$,

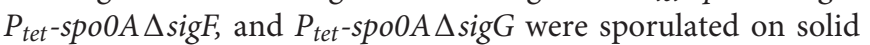
media in the presence or absence of ATc and analyzed by microscopy following membrane and DNA staining (Figure 5). When induced, both the sigE and sigF mutants were blocked at the asymmetric division stage, revealing abortive disporic cells and occasionally multiple, closely located polar septa. Given the apparent lack of DNA staining in compartments created by these multiple septa, the observed staining pattern could equally be the result of membrane delamination between the forespore and the mother cell. Additionally, sigF mutant forespores showed distinct bulging. While cells of the sigG mutant were able to complete engulfment, they did not proceed further in morphogenesis and the resulting forespores were often misshapen and dislodged, resting at an angle to the longitudinal axis of the cell. As expected, strains grown in the absence of ATc did not initiate sporulation (Figure 5). These observations are largely consistent with previous work characterizing the morphology of sporulationspecific sigma factor mutants created using ClosTron insertional 

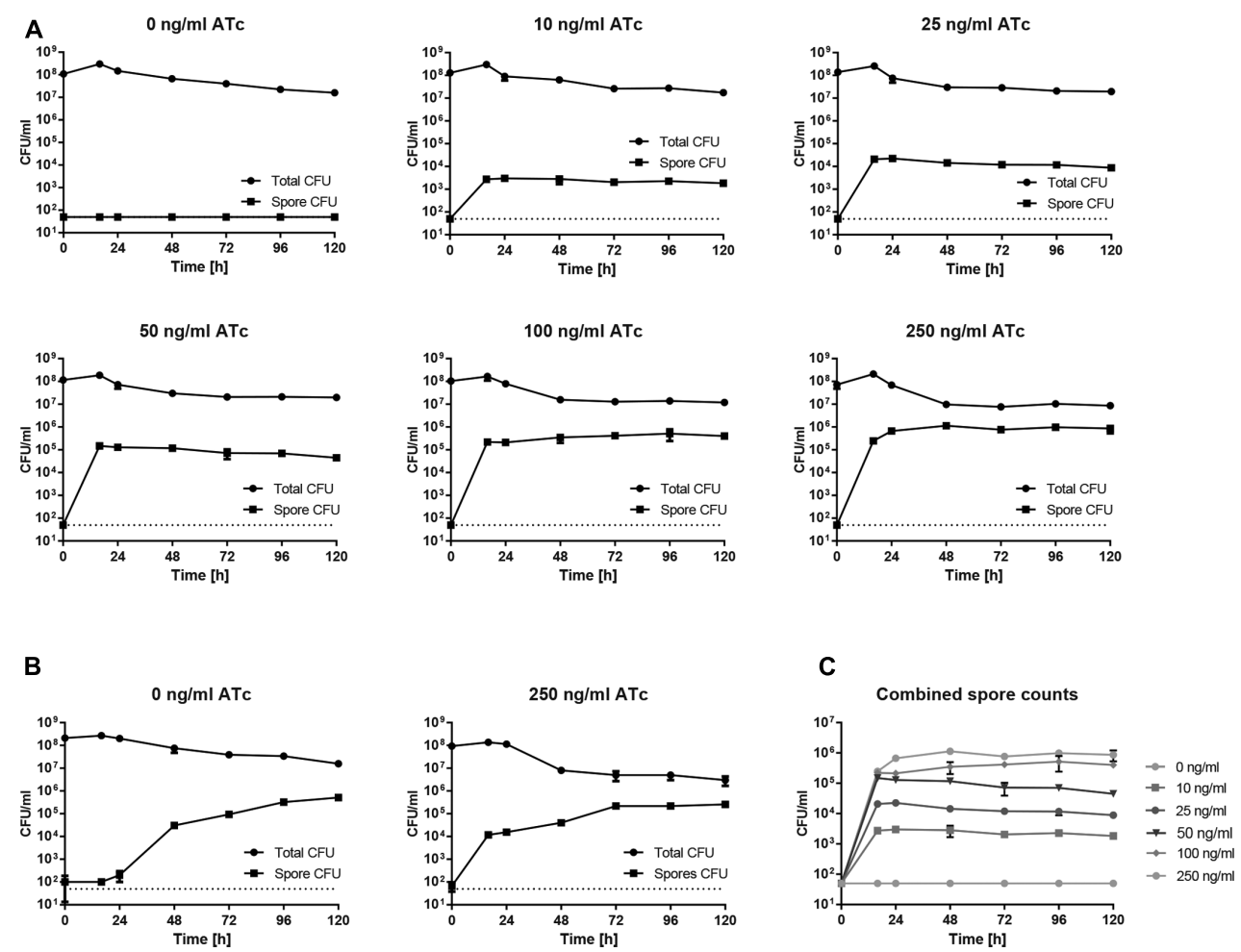

FIGURE 3 | Dose-dependent increase in sporulation efficiency in $P_{\text {tet }}$-spoOA. $P_{\text {tet }}$-spoOA (A) or WT (B) were grown to OD 0.8 and sub-cultured 1:10,000 in fresh $\mathrm{BHIS}$ supplemented with the concentration of ATc indicated, or $1 \%$ ethanol $(0 \mathrm{ng} / \mathrm{ml}$ ATc). Samples were removed at the indicated time points post-inoculation, serially diluted in sterile, pre-reduced PBS and plated onto BHIS agar supplemented with $0.1 \%$ Tch with (spore CFU) or without (total CFU) prior heat-treatment at $70^{\circ} \mathrm{C}$ for $30 \mathrm{~min}$. Colonies were enumerated after $24 \mathrm{~h}$ of incubation under anaerobic conditions. (C) Combined $P_{\text {tet }}$-spoOA counts showing a dose dependent increase in sporulation efficiency. Data presented as means \pm SD from three technical replicates. Each result is representative of experiments performed in at least biological triplicate. Dotted line represents the limit of detection.

mutagenesis (Pereira et al., 2013) and demonstrate the potential of using $P_{\text {tet }}-s p o 0 A$ in studies that would benefit from more control over the sporulation process.

\section{Use of $P_{\text {tet }}$-spoOA in the Mouse Model of Infection}

The spo0A regulatory network co-ordinates multiple aspects of the $C$. difficile life cycle, including toxin production and the resulting virulence of a given strain. Deakin et al. (2012) reported that a R20291 $\triangle$ spo0A strain (ribotype 027) produced more toxin in vitro and caused more severe disease than WT in a murine model of infection, while being deficient in persistence and transmission. More recent studies however, suggest that this effect is not consistent across all $C$. difficile strains (Mackin et al., 2013). While Spo0A was confirmed to be a negative regulator of toxin production in ribotype 027 strains, no such effect was observed in ribotype 012 , suggesting that Spo0A could differentially regulate toxin production between phylogenetically distinct strains of $C$. difficile (Mackin et al., 2013). The exact mechanisms of the increased virulence of some spo0A mutants are not yet known, but the explanation is most likely to be multi-faceted. In B. subtilis, Spo0A does not have an equal affinity for all genes within the spo0A regulon, leading to a programmed change in gene expression with sporulation-specific genes not activated until high levels of Spo0A have accumulated (Fujita et al., 2005). An inducible system that offers titratable control over Spo0A levels provides an opportunity to ask more nuanced questions about the role of Spo0A in the infectious process in a manner that the $\triangle$ spo0A mutant does not. Previous studies have demonstrated the potential for ATc induction of bacterial gene expression in mouse models (Ji et al., 2001; Kotula et al., 2014). Therefore, having characterized the $P_{\text {tet }}$-spo0A strain in vitro, we asked whether the inducible system would also work in vivo.

In our study, 12 mice pre-treated with clindamycin were infected with $1 \times 10^{7} \mathrm{CFU}$ from an overnight culture of $P_{\text {tet }}$-spo0A grown without ATc. Six mice received $0.1 \mathrm{mg} / \mathrm{ml}$ ATc in their drinking water, with the remaining six receiving $1 \%$ $(\mathrm{v} / \mathrm{v})$ ethanol as a negative control. Feces were collected at 24, 72 , and $120 \mathrm{~h}$ post-infection, and total and heat-resistant CFUs measured (Figure 6). No statistically significant difference was found for total cell counts between the $\mathrm{ATc}^{-}$and $\mathrm{ATc}^{+}$groups until $120 \mathrm{~h}$ after infection, indicating successful infection of both groups. For the group of mice that did not receive ATc in their drinking water, no spores could be detected by enumeration of heat resistant colonies, indicating a failure to sporulate within the mouse. In contrast, for the group of mice that received 

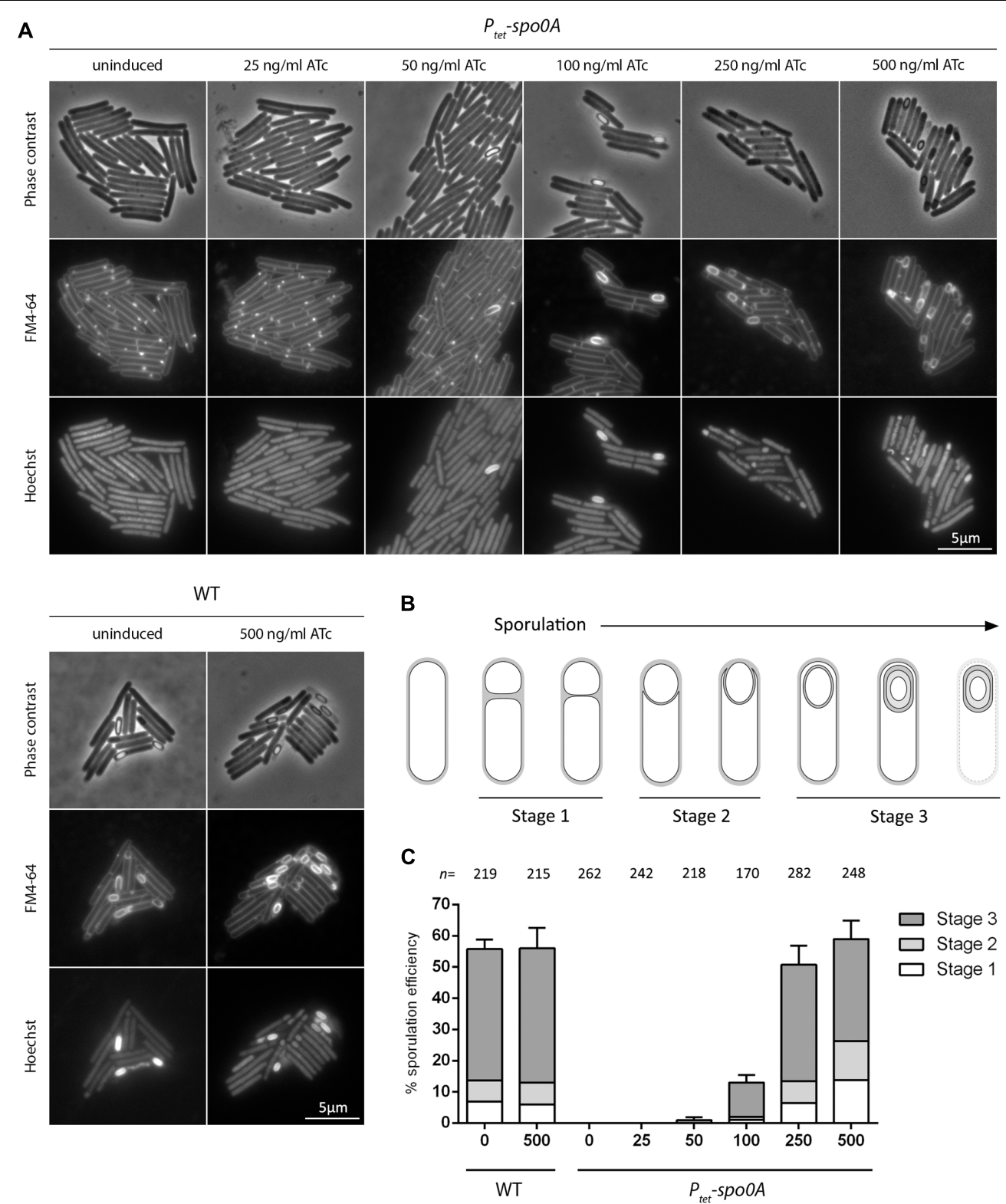

FIGURE 4 | Analysis of $P_{\text {tet }}$-SpoOA sporulation dynamics. (A) WT and $P_{\text {tet }}$-spo0A cultures were harvested after $14 \mathrm{~h}$ of growth on $70: 30$ agar supplemented with ATc as indicated. Cells were stained with FM4-64 and Hoechst 33258 and analyzed by phase contrast and fluorescence microscopy. Scale bar corresponds to $5 \mu \mathrm{m}$. (B) Schematic representation of the sporulation process indicating stages corresponding to asymmetric cell division (stage 1), engulfment (stage 2) and spore

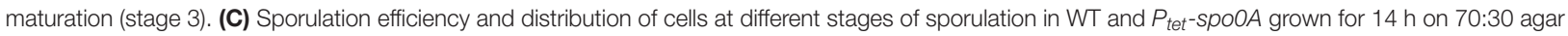
supplemented with ATc as indicated. Sporulation efficiency is expressed as the percentage ratio of cells at a given stage of sporulation to total cells counted. Data reported as means \pm SD from 10 random fields of view. The total number of cells counted (n) is indicated above each column on the graph.

$0.1 \mathrm{mg} / \mathrm{ml} \mathrm{ATc}$ in their drinking water, heat-resistant CFUs were detected $24 \mathrm{~h}$ post-infection, steadily increasing until the final time-point taken $120 \mathrm{~h}$ post-infection. To confirm the identity of the isolated strains post-infection, five colonies from each group were selected at random and screened for $P_{t e t}-s p o 0 A$ by PCR. As shown in Supplementary Figure S2, all strains were found to carry $P_{\text {tet }}$-spo0A. Thus, the $P_{\text {tet }}$ promoter does not become activated in vivo, rendering $P_{\text {tet }}-s p o 0 A$ asporogenous. Furthermore, ATc can be used to control the $P_{\text {tet }}$ system in vivo.
Therefore, this approach will permit the study of the role of Spo0A and sporulation/germination kinetics in infection with a level of control not possible with previously used systems.

\section{DISCUSSION}

Sporulation is a key contributing factor in establishing C. difficile as a leading nosocomial pathogen in the developed world. 


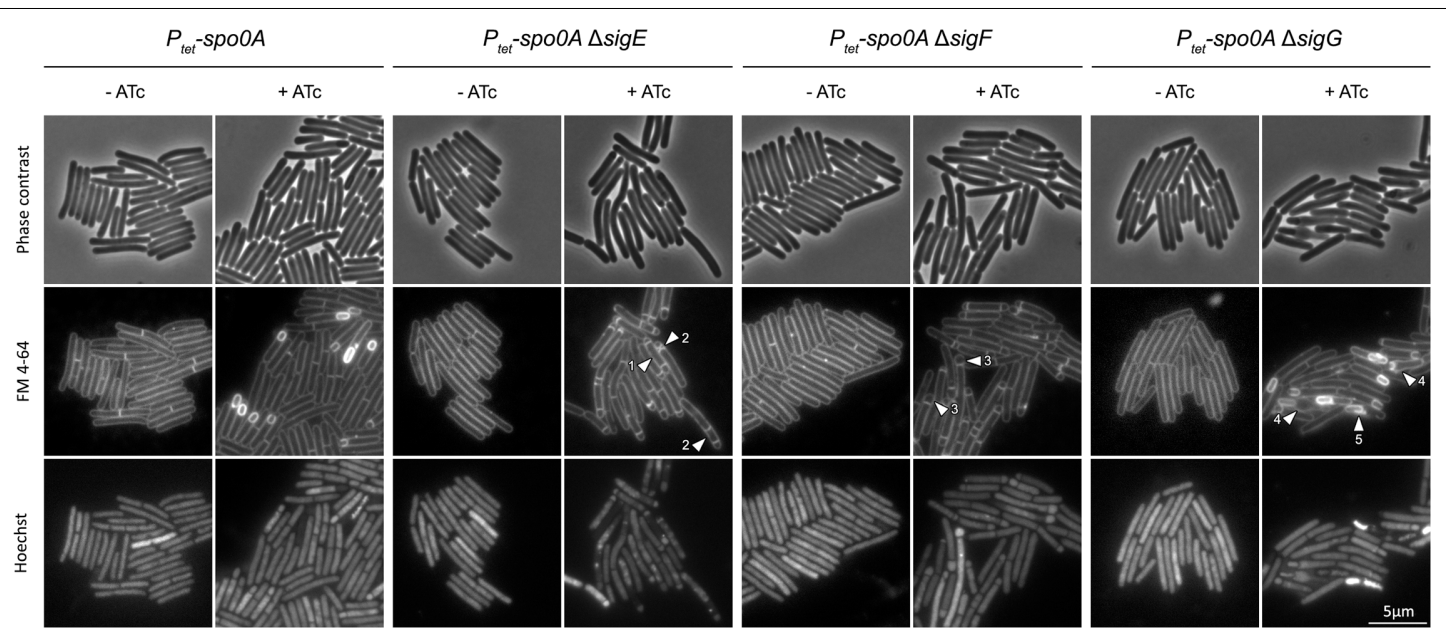

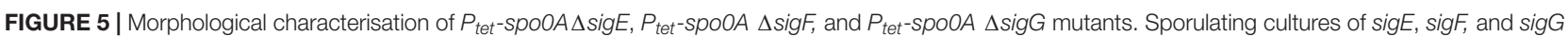
mutants created in the $P_{\text {tet }}$-SPOOA background were harvested after $14 \mathrm{~h}$ of growth on 70:30 agar supplemented with ATc as indicated. Cells were stained with FM4-64 and Hoechst 33258 and analyzed by phase contrast and fluorescence microscopy. Arrows indicate: (1) abortive diasporic forms, (2) multiple polar septa, (3) forespore bulging, (4) mother cell bulging and (5) dislodged sporangia resting at an angle to the longitudinal axis of the cell. Scale bar corresponds to $5 \mu \mathrm{m}$.
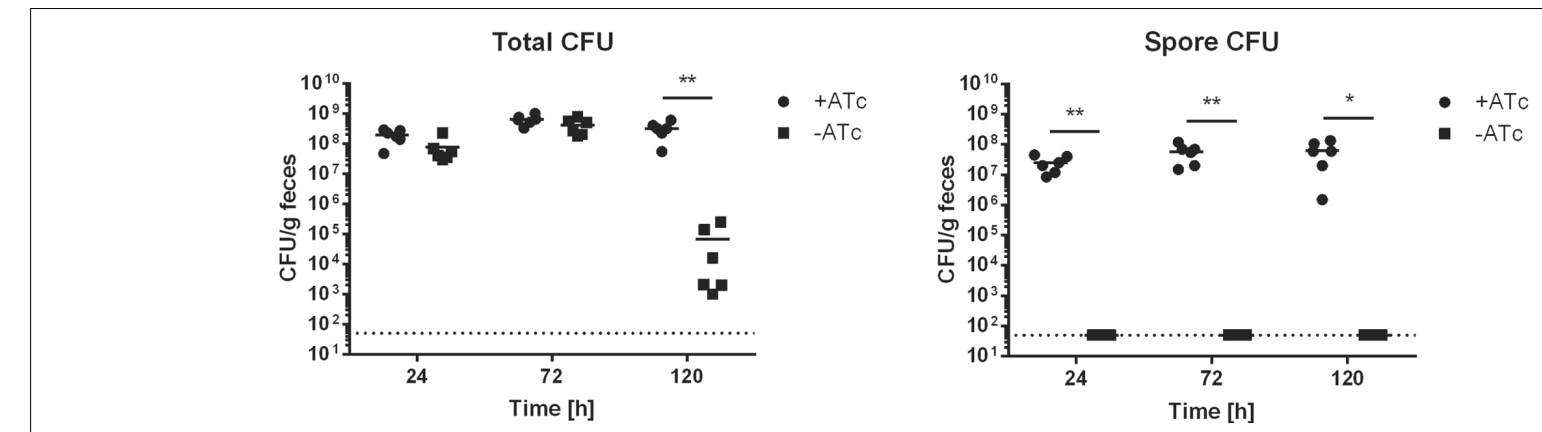

FIGURE 6 | Use of the $P_{\text {tet }}$ inducible system in vivo. 12 mice were treated with clindamycin to disrupt the natural flora. All mice were subsequently infected with the same culture of $P_{\text {tet }}$-SpOOA grown overnight without ATc (infectious dose $=1 \times 10^{7} \mathrm{CFU}$ ). The mice were then split into two groups of 6 , one of which received $1 \%$ ethanol in their drinking water, and the other $100 \mu \mathrm{g} / \mathrm{ml}$ ATc. Feces were collected at the time indicated post-infection and enumerated for total and heat-resistant CFUs. Data plotted for each animal individually with mean indicated $\left({ }^{*} p \leq 0.05 ;{ }^{* *} p \leq 0.01\right.$ by a multiple Student's $t$-test using the Holm-Sidak method). Dotted line represents the limit of detection.

Studies carried out in B. subtilis have undoubtedly provided a useful basis for study of sporulation in Clostridial species, but it has become clear that there are several key differences in the sporulation process which make extrapolating results difficult. These differences are most apparent at the stage of entry into sporulation, governed in Bacilli by a phosphorelay system that is absent in Clostridial species. It is presumed that the phosphorelay system provides tighter regulation over the commitment to sporulate, but this premise remains unproven and the consequences of the lack of such a system remain unclear. Another aspect of the $C$. difficile life cycle that is uniquely affected by $\mathrm{Spo} 0 \mathrm{~A}$ is toxin production, a phenotype that cannot be studied in a non-toxigenic species such as $B$. subtilis. Additionally, Spo0A regulates other lifestyle changes in a manner that can vary from species to species and even between strains. Thus, although Spo0A is the master regulator of sporulation, it is also an important instigator of transcriptional changes that might help evade the need to sporulate, such as cannibalism or biofilm formation.

Under laboratory conditions, $C$. difficile sporulation efficiency is low compared to B. subtilis (Burns and Minton, 2011), a technical hurdle to its study that is exacerbated by the high heterogeneity and the current inability to synchronize a sporulating culture. Furthermore, as illustrated in studies of B. subtilis, the study of spooA-null mutants does not permit recognition of the importance of incremental increases in the level of Spo0A, leading to more nuanced Spo0A-mediated phenotypes to be overlooked. An over-simplification of the intricate Spo0A network is perhaps one reason for conflicting results regarding Spo0A-mediated phenotypes in the literature. In this study, we report the generation of an inducible spo0 A system, as schematically detailed in Figure 1A, in an attempt to improve sporulation efficiency, investigate the possibility of 
achieving a more homogenous culture, and test spo0A-titratable phenotypes.

In WT $C$. difficile cultures, heat-resistant CFUs are typically observed 8-12 h after reaching stationary phase (Pereira et al., 2013) and the sporulation process can take up to 5 days to reach equilibrium (Burns and Minton, 2011). We have demonstrated that inducing spo0A expression significantly reduces the amount of time necessary to reach maximum spore titers. Analysis of the cultures by fluorescence microscopy indicated that sporulation proceeds similarly to WT even when induction leads to faster completion of sporulation, but transmission electron microscopy would be required to rule out any changes to the fine structure of the spore. These results demonstrates that induction of Spo0A is sufficient to drive sporulation and, at least to some degree, overrides the need for external stimulus. Therefore, the $P_{\text {tet- }}-s p 00 A$ strain is a useful background for generating mutants in genes involved in other stages of the sporulation pathway, as illustrated by construction of sporulation-specific sigma factor mutants. Furthermore, the $P_{\text {tet }}$ promotor removes spo0A from its normal regulatory network; this allows the maintenance of high levels of spo0A expression when mutation of a gene in the sporulation network might otherwise serve to downregulate $s p o 0 A$ and potentially mask phenotypes of interest.

Our finding that accelerated accumulation of Spo0A correlates with more rapid sporulation contrasts with studies performed in B. subtilis, where accelerated accumulation is detrimental to sporulation (Vishnoi et al., 2013). We found no evidence that fast accumulation to elevated levels of Spo0A has any detrimental effect on sporulation, indicating that a gradual buildup of Spo0A is not important to the sporulation process itself in C. difficile. In B. subtilis, it has been suggested that the reduction in sporulation efficiency upon artificial induction of Spo0A resulted from premature repression of DivIVA, which is involved in chromosome segregation during the earlier stages of sporulation but downregulated via Spo0A in the later stages of sporulation (Vishnoi et al., 2013). Existing transcriptomic and proteomic studies of $C$. difficile $\triangle$ spo0A have not found a statistically significant regulation of DivIVA by Spo0A (Pettit et al., 2014; Charlton et al., 2015), but the $P_{\text {tet }}$-spo0A strain will allow a more in-depth study of this connection, as well as investigations into the potential existence of high and low spo0A regulons more generally. It has been previously proposed that the phosphorelay system present in B. subtilis is responsible for the gradual build-up of Spo0A (Fujita and Losick, 2005) and our finding would be in line with this notion.

Finally, we demonstrated that the inducible system works in a mouse model of infection, with $P_{\text {tet }}-s p o 0 A$ remaining asporogenous in the absence of ATc in drinking water but sporulating in its presence. Although beyond the scope of this work, $P_{\text {tet }}-s p o 0 A$ could be used to investigate germination rates and efficiency in vivo, following infection with spores produced by ATc induction in vitro which will be unable to re-sporulate in vivo. Furthermore, we envisage that administration of ATc at different doses relative to mouse weight will allow dosedependent phenotypes to be studied during infection. This paves the way for more detailed study into the role of Spo0A in virulence, and to delineate the relative importance of the many Spo0A related factors involved. The $P_{\text {tet }}-s p o 0 A$ strain described here is therefore a novel tool to study sporulation in $C$. difficile in a much more robust and controllable way, that will allow us to gain unique insights into the mechanisms involved in spore formation and related phenotypes, as well as the role of Spo0A in pathways leading to different lifecycle choices.

\section{MATERIALS AND METHODS}

\section{Bacterial Strains and Growth Conditions}

Escherichia coli strains were grown in LB-Miller supplemented where necessary with chloramphenicol (15 $\mu \mathrm{g} / \mathrm{ml})$ and/or kanamycin $(50 \mu \mathrm{g} / \mathrm{ml})$. C. difficile strains were cultured statically in BHIS (37 $\mathrm{g}$ brain heart infusion, $5 \mathrm{~g}$ yeast extract and $1 \mathrm{~g}$ L-cysteine per liter), SM (90 g Bacto peptone, $5 \mathrm{~g}$ proteose peptone, $1 \mathrm{~g} \mathrm{NH} \mathrm{SO}_{4}, 1.5 \mathrm{~g}$ Tris base) or a 70:30 mixture of SM and BHIS as indicated, supplemented where necessary with thiamphenicol $(15 \mu \mathrm{g} / \mathrm{ml})$, D-cycloserine $(250 \mu \mathrm{g} / \mathrm{ml})$, 5 -fluorocytosine (FC) $(50 \mu \mathrm{g} / \mathrm{ml})$ or anhydrotetracycline (ATc) (5-500 ng/ml). For plate cultures, media were solidified with $1.5 \%$ $(\mathrm{w} / \mathrm{v})$ agar. All C. difficile strains were grown and maintained at $37^{\circ} \mathrm{C}$ in a Don Whitley DG250 anaerobic workstation under anaerobic conditions $\left(10 \% \mathrm{H}_{2}, 10 \% \mathrm{CO}_{2}, 80 \% \mathrm{~N}_{2}\right.$ ) (Don Whitley Scientific). A detailed list of strains used in this study is provided in Supplementary Table S1.

\section{Construction of $\boldsymbol{P}_{\text {tet }}$-spoOA Mutant}

To generate plasmid pSEW200, the following DNA fragments were PCR-amplified using KOD Hot Start polymerase (Novagen). The pMTL-SC7315 plasmid backbone (Cartman et al., 2012) was linearized by inverse PCR using primers 103 and 104. 1,200 bp upstream and downstream spo0A homology arms were amplified from $C$. difficile 630 genomic DNA, using primer pairs 155-156 and 159-160, respectively. Tet regulatory elements were amplified from pRPF185 (Fagan and Fairweather, 2011) using primer pair 157-158. The resulting fragments were ligated using Gibson assembly (NEB) as per manufacturer's instructions. pSEW200 was transformed into E. coli CA434 and then conjugated into either $C$. difficile 630 or $C$. difficile $630 \Delta \mathrm{erm}$ as described previously (Purdy et al., 2002). Resulting colonies were restreaked twice onto fresh BHIS agar plates supplemented with thiamphenicol and D-cycloserine to counter-select for E. coli. Allelic exchange was performed as described previously (Cartman et al., 2012), with the slight modification of colonies being restreaked three times onto $C$. difficile Defined Medium (CDDM) (Karasawa et al., 1995) agar plates supplemented with FC $(50 \mu \mathrm{g} / \mathrm{ml})$ prior to patch-plating on BHIS agar plates supplemented with thiamphenicol to screen for plasmid excision. FC-resistant, thiamphenicol-sensitive clones were screened by PCR to separate mutants from WT revertants using primer pair 217-218. A detailed list of plasmids and primers used in this study is provided in Supplementary Tables S2 and S3, respectively. 


\section{Construction of sigEFG Mutants}

sigEFG mutants were created in the $630 \Delta \mathrm{erm} P_{\text {tet }}-$ spo0A background via allele-coupled exchange as described previously (Cartman et al., 2012). Briefly, the pMTL-SC7315 plasmid backbone was linearized by inverse PCR using primers 103 and 104. $900 \mathrm{bp}$ upstream and downstream homology arms were PCR amplified from $C$. difficile 630 genomic DNA, using primer pairs 161-162 and 163-164 (sigE); 167-168 and 169-170 (sigF); 174-175 and 176-177 (sigG) respectively. The resulting fragments were ligated using Gibson assembly (NEB) as per manufacturer's instructions yielding pMLD126 (sigE), pMLD127 $(s i g F)$, and pMLD128 (sigG). All plasmids were transformed into E. coli CA434 and conjugated into C. difficile $630 \Delta \mathrm{erm}$ $P_{\text {tet }}$-spo0A as described previously (Purdy et al., 2002). Resulting colonies were restreaked twice onto fresh BHIS agar plates supplemented with thiamphenicol and D-cycloserine to counterselect for E. coli. These were then restreaked onto CDMM agar plates supplemented with FC $(50 \mu \mathrm{g} / \mathrm{ml})$ before patch-plating on BHIS agar supplemented with thiamphenicol to screen for plasmid excision. FC-resistant, thiamphenicol-sensitive clones were screened by PCR to separate mutants from WT revertants using primer pairs 165-166 (sigE), 171-172 (sigF), and 177-178 $(\operatorname{sigG})$.

\section{RNA Extraction and Quantitative Reverse Transcription PCR Analysis (qRT-PCR)}

RNA was extracted from $C$. difficile cultures grown in BHIS to early stationary phase $\left(\mathrm{OD}_{600} \sim 0.8\right)$ using the Total RNA Purification Kit (Norgen Biotek, Corp.) according to the manufacturer's instructions. Purified RNA was DNasetreated using the Turbo DNA-free Kit (Life Technologies). First strand synthesis was performed on $1 \mu \mathrm{g}$ of RNA using the SuperScript III VILO cDNA Synthesis Kit (Invitrogen) according to the manufacturer's instructions. 50 ng of cDNA per reaction was used to perform qRT-PCR analysis using the SensiFast SYBR No-ROX Kit (Bioline) on a RotorGene 6000 instrument (Corbett Research). cDNA synthesis reactions containing no reverse transcriptase were included to control for genomic contamination. Primers for qRT-PCR analysis were designed using PrimerQuest (Integrated DNA Technologies), and primer efficiencies were calculated for each primer set prior to use. qRT-PCR was performed in technical triplicate for each cDNA sample and primer pair and on cDNA isolated from a minimum of two biological replicates. Results were calculated by the comparative cycle threshold method (Schmittgen and Livak, 2008) and normalized to the rpoC transcript. Results are presented as the means and standard deviations of the means and a two-tailed Student's $t$-test was performed to determine statistical significance using GraphPad Prism 6.

\section{Sporulation Assays}

C. difficile cultures were grown in BHIS broth to logarithmic growth phase $\left(\mathrm{OD}_{600} \sim 0.6\right)$, diluted $1: 10,000$ in fresh broth, and grown overnight to stationary phase. This allowed synchronization of growth and minimized the carry-over of spores from initial cultures. Total and heat-resistant CFUs were then enumerated at $24 \mathrm{~h}$ intervals for 5 days. To this end, at each time point serial 10 -fold dilutions were prepared in pre-reduced PBS and $20 \mu \mathrm{l}$ were spotted in triplicate onto BHIS agar supplemented with $0.1 \%(\mathrm{w} / \mathrm{v})$ sodium taurocholate. To determine the number of spores, samples were incubated at $70^{\circ} \mathrm{C}$ for $30 \mathrm{~min}$ before spotting onto plates. Colonies were enumerated after $24 \mathrm{~h}$ incubation in an anaerobic cabinet.

\section{Microscopy}

$0.5 \mathrm{ml}$ samples from cultures grown in SM broth were harvested by centrifugation $(2 \min$ at $4,000 \times g) 14 \mathrm{~h}$ after inoculation. Cells were washed with $1 \mathrm{ml}$ of PBS, resuspended in $100 \mu \mathrm{l}$ of PBS and spotted onto $1.5 \%(\mathrm{w} / \mathrm{v})$ agarose pads supplemented with a lipophilic steryl membrane dye: $\mathrm{N}-(3-$ triethylammoniumprpyl)24-( $p$-diethylaminophenyl-hexatrienyl) pyridinium dibromide (FM4-64; Invitrogen; $1 \mu \mathrm{g} / \mathrm{ml}$ ), a DNA dye: $\quad 2$-(4-hydroxyphenyl)-5-(4-methyl-1-piperazinyl)-2,5'bi- $1 H$-benzimidazole trihydrochloride hydrate, bisBenzimide (Hoechst 33258, Thermo Fisher; $1 \mu \mathrm{g} / \mathrm{ml}$ ) and $0.5 \%(\mathrm{v} / \mathrm{v})$ di-methylsulfoxide (DMSO). Images were captured using the Metamorph software package on a Nikon Ti microscope and were processed using ImageJ (Schindelin et al., 2012; Schneider et al., 2012).

\section{Western Blotting}

To prepare C. difficile whole cell lysates, $0.5 \mathrm{ml}$ samples from cultures grown overnight in BHIS to stationary phase were harvested by centrifugation $(2 \mathrm{~min}$ at $4,000 \times g)$, washed with PBS, resuspended to $\mathrm{OD}_{600}=10$ in sample buffer and boiled for $10 \mathrm{~min}$. S-layer extracts were prepared using low $\mathrm{pH}$ glycine as previously described (Calabi et al., 2001). SDS-PAGE was carried out according to standard protocols. For immunoblot analysis, proteins were transferred to nitrocellulose membranes using the Trans-blot Turbo system (Bio-rad) according to the manufacturer's instructions. Membranes were blocked in $3 \%(\mathrm{w} / \mathrm{v})$ skimmed milk in PBS before probing with rabbit anti-Spo0A antibody at 1:5,000 or chicken anti-FliC at 1:100,000. Primary antibodies were detected using horseradish peroxidase (HRP)-conjugated goat anti-rabbit $\operatorname{IgG}(\mathrm{H}+\mathrm{L})$ antibody at 1:2,500 (Promega) or goat anti-chicken IgY antibody at 1:10,000 (Novex, Life Technologies) and Clarity Western ECL substrate (Bio-rad).

\section{Animal Studies}

12 C57BL/6 mice aged 6-7 weeks (Charles River) were pretreated with drinking water containing $250 \mathrm{mg} / \mathrm{ml}$ clindamycin for 7 days. Clindamycin treatment was then removed for $24 \mathrm{~h}$, after which the mice were infected by oral gavage with $1 \times 10^{7}$ CFU $\mathrm{P}_{\text {tet }}-$ spo0A grown for $16 \mathrm{~h}$ in TYG (3\% w/v Bacto tryptose, $2 \% \mathrm{w} / \mathrm{v}$ yeast, $0.5 \% \mathrm{w} / \mathrm{v}$ glucose). The mice were split into two groups of 6 , each receiving either $0.1 \mathrm{mg} / \mathrm{ml} \mathrm{ATc}$ or $1 \%(\mathrm{v} / \mathrm{v})$ ethanol in drinking water, refreshed every $24 \mathrm{~h}$. Fresh feces were collected and suspended in sterilized distilled water to $0.2 \mathrm{~g} / \mathrm{ml}$. For heat-treated counts, the suspension was treated at $70^{\circ} \mathrm{C}$ for 
$30 \mathrm{~min} .20 \mu \mathrm{l}$ of suspension was plated in technical triplicate on Chrome ID plates (BioMerieux) and CFU/g fecal matter enumerated following $16 \mathrm{~h}$ growth.

\section{ETHICS STATEMENT}

All animal work was performed under the United Kingdom Home Office project license PPL 70/8276 and with approval from the Royal Holloway University of London Ethics Committee.

\section{AUTHOR CONTRIBUTIONS}

MD and SW designed the study, developed the methodology, collected and analyzed the data, wrote and revised the manuscript; $\mathrm{SH}$ and $\mathrm{HH}$ collected the data; $\mathrm{PS}$ and SC designed the study, analyzed the data, revised the manuscript.

\section{REFERENCES}

Al-Hinai, M. A., Jones, S. W., and Papoutsakis, E. T. (2015). The Clostridium sporulation programs: diversity and preservation of endospore differentiation. Microbiol. Mol. Biol. Rev. 79, 19-37. doi: 10.1128/MMBR.00025-14

Burns, D. A., Heap, J. T., and Minton, N. P. (2010). The diverse sporulation characteristics of Clostridium difficile clinical isolates are not associated with type. Anaerobe 16, 618-622. doi: 10.1016/j.anaerobe.2010.10.001

Burns, D. A., and Minton, N. P. (2011). Sporulation studies in Clostridium difficile. J. Microbiol. Methods 87, 133-138. doi: 10.1016/j.mimet.2011.07.017

Calabi, E., Ward, S., Wren, B., Paxton, T., Panico, M., Morris, H., et al. (2001). Molecular characterization of the surface layer proteins from Clostridium difficile. Mol. Microbiol. 40, 1187-1199. doi: 10.1046/j.1365-2958.2001. 02461.x

Cartman, S. T., Kelly, M. L., Heeg, D., Heap, J. T., and Minton, N. P. (2012). Precise manipulation of the Clostridium difficile chromosome reveals a lack of association between tcdC genotype and toxin production. Appl. Environ. Microbiol. 78, 4683-4690. doi: 10.1128/AEM.00249-12

Charlton, T. M., Kovacs-Simon, A., Michell, S. L., Fairweather, N. F., and Tate, E. W. (2015). Quantitative lipoproteomics in Clostridium difficile reveals a role for lipoproteins in sporulation. Chem. Biol. 22, 1562-1573. doi: 10.1016/ j.chembiol.2015.10.006

Dawson, L. F., Valiente, E., Donahue, E. H., Birchenough, G., and Wren, B. W. (2011). Hypervirulent Clostridium difficile PCR-ribotypes exhibit resistance to widely used disinfectants. PLOS ONE 6:e25754. doi: 10.1371/journal.pone. 0025754

Deakin, L. J., Clare, S., Fagan, R. P., Dawson, L. F., Pickard, D. J., West, M. R., et al. (2012). The Clostridium difficile spo0A gene is a persistence and transmission factor. Infect. Immun. 80, 2704-2711. doi: 10.1128/IAI.00147-12

Dethlefsen, L., Huse, S., Sogin, M. L., and Relman, D. A. (2008). The pervasive effects of an antibiotic on the human gut microbiota, as revealed by deep $16 \mathrm{~S}$ rRNA sequencing. PLOS Biol. 6:e280. doi: 10.1371/journal.pbio.0060280

Dubberke, E. R., and Olsen, M. A. (2012). Burden of Clostridium difficile on the healthcare system. Clin. Infect. Dis. 55(Suppl. 2), S88-S92. doi: 10.1093/cid/ cis335

Edwards, A. N., Nawrocki, K. L., and Mcbride, S. M. (2014). Conserved oligopeptide permeases modulate sporulation initiation in Clostridium difficile. Infect. Immun. 82, 4276-4291. doi: 10.1128/IAI.02323-14

Edwards, A. N., Tamayo, R., and Mcbride, S. M. (2016). A novel regulator controls Clostridium difficile sporulation, motility and toxin production. Mol. Microbiol. 100, 954-971. doi: $10.1111 / \mathrm{mmi} .13361$

\section{FUNDING}

This work was supported by grants from the MRC (DPFS MR/K015354/1) and European Union (HEALTH-F3-2013601810) to SC. MD is supported by an MRC NIRG (MR/M000923/1) awarded to PS.

\section{ACKNOWLEDGMENT}

We would like to thank Prof. Glen D. Armstrong for providing the anti-FliC antibodies and Dr. Aimee Shen for providing the anti-Spo0A antibodies.

\section{SUPPLEMENTARY MATERIAL}

The Supplementary Material for this article can be found online at: http://journal.frontiersin.org/article/10.3389/fmicb. 2017.01793/full\#supplementary-material

Fagan, R. P., and Fairweather, N. F. (2011). Clostridium difficile has two parallel and essential Sec secretion systems. J. Biol. Chem. 286, 27483-27493. doi: 10.1074/ jbc.M111.263889

Fimlaid, K. A., Bond, J. P., Schutz, K. C., Putnam, E. E., Leung, J. M., Lawley, T. D., et al. (2013). Global analysis of the sporulation pathway of Clostridium difficile. PLOS Genet. 9:e1003660. doi: 10.1371/journal.pgen.1003660

Fujita, M., Gonzalez-Pastor, J. E., and Losick, R. (2005). High- and low-threshold genes in the Spo0A regulon of Bacillus subtilis. J. Bacteriol. 187, 1357-1368. doi: 10.1128/JB.187.4.1357-1368.2005

Fujita, M., and Losick, R. (2005). Evidence that entry into sporulation in Bacillus subtilis is governed by a gradual increase in the level and activity of the master regulator Spo0A. Genes Dev. 19, 2236-2244. doi: 10.1101/gad.1335705

Heap, J. T., Ehsaan, M., Cooksley, C. M., Ng, Y. K., Cartman, S. T., Winzer, K., et al. (2012). Integration of DNA into bacterial chromosomes from plasmids without a counter-selection marker. Nucleic Acids Res. 40:e59. doi: 10.1093/nar/gkr1321

Ji, Y., Zhang, B., Van Horn, S. F., Warren, P., Woodnutt, G., Burnham, M. K., et al. (2001). Identification of critical staphylococcal genes using conditional phenotypes generated by antisense RNA. Science 293, 2266-2269. doi: 10.1126/ science.1063566

Jiang, M., Grau, R., and Perego, M. (2000a). Differential processing of propeptide inhibitors of Rap phosphatases in Bacillus subtilis. J. Bacteriol. 182, 303-310.

Jiang, M., Shao, W., Perego, M., and Hoch, J. A. (2000b). Multiple histidine kinases regulate entry into stationary phase and sporulation in Bacillus subtilis. Mol. Microbiol. 38, 535-542.

Karasawa, T., Ikoma, S., Yamakawa, K., and Nakamura, S. (1995). A defined growth medium for Clostridium difficile. Microbiology 141(Pt 2), 371-375. doi: 10.1099/ 13500872-141-2-371

Kotula, J. W., Kerns, S. J., Shaket, L. A., Siraj, L., Collins, J. J., Way, J. C., et al. (2014). Programmable bacteria detect and record an environmental signal in the mammalian gut. Proc. Natl. Acad. Sci. U.S.A. 111, 4838-4843. doi: 10.1073/ pnas.1321321111

Lawley, T. D., Clare, S., Deakin, L. J., Goulding, D., Yen, J. L., Raisen, C., et al. (2010). Use of purified Clostridium difficile spores to facilitate evaluation of health care disinfection regimens. Appl. Environ. Microbiol. 76, 6895-6900. doi: 10.1128/AEM.00718-10

Lawley, T. D., and Walker, A. W. (2013). Intestinal colonization resistance. Immunology 138, 1-11. doi: 10.1111/j.1365-2567.2012.03616.x

Mackin, K. E., Carter, G. P., Howarth, P., Rood, J. I., and Lyras, D. (2013). Spo0A differentially regulates toxin production in evolutionarily diverse strains of Clostridium difficile. PLOS ONE 8:e79666. doi: 10.1371/journal.pone.00 79666 
Molle, V., Fujita, M., Jensen, S. T., Eichenberger, P., Gonzalez-Pastor, J. E., Liu, J. S., et al. (2003). The Spo0A regulon of Bacillus subtilis. Mol. Microbiol. 50, 1683-1701. doi: 10.1046/j.1365-2958.2003.03818.x

Nawrocki, K. L., Edwards, A. N., Daou, N., Bouillaut, L., and Mcbride, S. M. (2016). CodY-dependent regulation of sporulation in Clostridium difficile. J. Bacteriol. 198, 2113-2130. doi: 10.1128/JB.00220-16

Paredes, C. J., Alsaker, K. V., and Papoutsakis, E. T. (2005). A comparative genomic view of clostridial sporulation and physiology. Nat. Rev. Microbiol. 3, 969-978. doi: $10.1038 /$ nrmicro 1288

Pereira, F. C., Saujet, L., Tome, A. R., Serrano, M., Monot, M., CoutureTosi, E., et al. (2013). The spore differentiation pathway in the enteric pathogen Clostridium difficile. PLOS Genet. 9:e1003782. doi: 10.1371/journal. pgen. 1003782

Pettit, L. J., Browne, H. P., Yu, L., Smits, W. K., Fagan, R. P., Barquist, L., et al. (2014). Functional genomics reveals that Clostridium difficile Spo0A coordinates sporulation, virulence and metabolism. BMC Genomics 15:160. doi: 10.1186/1471-2164-15-160

Purdy, D., O’keeffe, T. A., Elmore, M., Herbert, M., Mcleod, A., Bokori-Brown, M., et al. (2002). Conjugative transfer of clostridial shuttle vectors from Escherichia coli to Clostridium difficile through circumvention of the restriction barrier. Mol. Microbiol. 46, 439-452. doi: 10.1046/j.1365-2958.2002.03134.x

Putnam, E. E., Nock, A. M., Lawley, T. D., and Shen, A. (2013). SpoIVA and SipL are Clostridium difficile spore morphogenetic proteins. J. Bacteriol. 195, 1214-1225. doi: 10.1128/JB.02181- 12

Rosenbusch, K. E., Bakker, D., Kuijper, E. J., and Smits, W. K. (2012). C. difficile 630Deltaerm Spo0A regulates sporulation, but does not contribute to toxin production, by direct high-affinity binding to target DNA. PLOS ONE 7:e48608. doi: 10.1371/journal.pone.0048608

Rupnik, M., Wilcox, M. H., and Gerding, D. N. (2009). Clostridium difficile infection: new developments in epidemiology and pathogenesis. Nat. Rev. Microbiol. 7, 526-536. doi: 10.1038/nrmicro2164

Schindelin, J., Arganda-Carreras, I., Frise, E., Kaynig, V., Longair, M., Pietzsch, T., et al. (2012). Fiji: an open-source platform for biological-image analysis. Nat. Methods 9, 676-682. doi: 10.1038/nmeth.2019

Schmittgen, T. D. and Livak, K. J. (2008). Analyzing real-time PCR data by the comparative $C_{T}$ method. Nat. Protoc. 3, 1101-1108. doi: 10.1038/nprot. 2008.73
Schneider, C. A., Rasband, W. S., and Eliceiri, K. W. (2012). NIH Image to ImageJ: 25 years of image analysis. Nat. Methods 9, 671-675. doi: 10.1038/nmeth.2089

Smits, W. K., Lyras, D., Lacy, D. B., Wilcox, M. H., and Kuijper, E. J. (2016). Clostridium difficile infection. Nat. Rev. Dis. Primers 2:16020. doi: 10.1038/nrdp. 2016.20

Sonenshein, A. L. (2000). Control of sporulation initiation in Bacillus subtilis. Curr. Opin. Microbiol. 3, 561-566. doi: 10.1016/S1369-5274(00)00141-7

Steiner, E., Dago, A. E., Young, D. I., Heap, J. T., Minton, N. P., Hoch, J. A., et al. (2011). Multiple orphan histidine kinases interact directly with Spo0A to control the initiation of endospore formation in Clostridium acetobutylicum. Mol. Microbiol. 80, 641-654. doi: 10.1111/j.1365-2958.2011.07608.x

Underwood, S., Guan, S., Vijayasubhash, V., Baines, S. D., Graham, L., Lewis, R. J., et al. (2009). Characterization of the sporulation initiation pathway of Clostridium difficile and its role in toxin production. J. Bacteriol. 191, 7296-7305. doi: 10.1128/JB.00882-09

Veening, J. W., Hamoen, L. W., and Kuipers, O. P. (2005). Phosphatases modulate the bistable sporulation gene expression pattern in Bacillus subtilis. Mol. Microbiol. 56, 1481-1494. doi: 10.1111/j.1365-2958.2005.04659.x

Vishnoi, M., Narula, J., Devi, S. N., Dao, H. A., Igoshin, O. A., and Fujita, M. (2013). Triggering sporulation in Bacillus subtilis with artificial two-component systems reveals the importance of proper Spo0A activation dynamics. Mol. Microbiol. 90, 181-194. doi: 10.1111/mmi.12357

Vohra, P., and Poxton, I. R. (2011). Efficacy of decontaminants and disinfectants against Clostridium difficile. J. Med. Microbiol. 60, 1218-1224. doi: 10.1099/ jmm.0.030288-0

Conflict of Interest Statement: The authors declare that the research was conducted in the absence of any commercial or financial relationships that could be construed as a potential conflict of interest.

Copyright (c) 2017 Dembek, Willing, Hong, Hosseini, Salgado and Cutting. This is an open-access article distributed under the terms of the Creative Commons Attribution License (CC BY). The use, distribution or reproduction in other forums is permitted, provided the original author(s) or licensor are credited and that the original publication in this journal is cited, in accordance with accepted academic practice. No use, distribution or reproduction is permitted which does not comply with these terms. 